\title{
Fitoderivati e dieta mediterranea: armi naturali contro la disfunzione mitocondriale e la progressione del danno renale cronico
}

\author{
Alessandra Dalla Gassa, Simona Granata, Nadia Antonucci, Antonio Lupo, Gianluigi Zaza \\ Unità di Nefrologia, Dipartimento di Medicina, Università di Verona, Verona
}

\begin{abstract}
Phytochemicals, diet and ckd-associated mitochondrial disfunction
Most recent evidence suggest that mitochondrial dysfunction plays a primary role in the development of chronic kidney disease and of severe associated comorbidities. This raises the possibility of considering mitochondria as a new possible therapeutic target in this important pathological condition.

A variety of natural extracts from plants in combination with conventional therapies and with a proper lifestyle could help doctors to reach the ambitious goal of preventing and slowing down chronic kidney disease progression.

Herbal remedies, known during the centuries in traditional medicines of countries all around the world such as black cumin, turmeric, green tea, olive and others are potential resources for extraction and purification of antioxidants and mitochondria-modulating substances, as many studies, some of which reported below, have partly already shown, also in nephrology. Many of them are characteristic of the Mediterranean diet; this diet appears effective in terms of prevention and prognosis in chronic kidney disease and related cardiovascular risk.

However, still extensive in vivo studies in animal models and subsequent trials are needed to provide more reliable information on nutraceuticals and phytoderivatives effectiveness in chronic kidney disease.

Several future strategies (i.e. pharmacogenomics and nutrigenomics) will strengthen these findings.
\end{abstract}

Keywords: Chronic kidney disease, Mediterranean diet, Mitochondria, Natural plant extracts, Oxidative stress

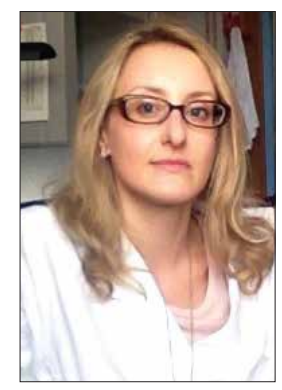

Alessandra Dalla Gassa

\section{Mitocondri e insufficienza renale cronica}

I mitocondri partecipano a numerose funzioni cellulari, tra cui l'omeostasi degli ioni e dell'eme e la sintesi di steroidi, il signaling del calcio e l'apoptosi. Il ruolo principale di questi organelli è di generare energia per il metabolismo cellulare attraverso il processo di fosforilazione ossidativa (OXPHOS).

Gli elettroni derivati dal metabolismo cellulare raggiungono i mitocondri attraverso due coenzimi, la nicotinammide adenina dinucleotide (NADH) e la flavina

Accepted: November 1, 2015

Published online: February 17, 2016

Indirizzo per la corrispondenza:

Dr. Gianluigi Zaza

Unità di Nefrologia, Dipartimento di Medicina

Università di Verona

Piazzale A. Stefani 1

37126 Verona

gianluigi.zaza@univr.it adenina dinucleotide (FADH2). Poi, subiscono un passaggio lungo tutta la catena di trasporto degli elettroni, che si compone di cinque complessi proteici situati nella membrana mitocondriale interna.

Gli elettroni passano attraverso complessi I, III e IV, grazie a un gradiente protonico generato dal trasporto di queste particelle sul lato esterno della membrana mitocondriale interna. Il complesso $\mathrm{V}$ investe, poi, l'energia derivata dal trasporto degli elettroni nella sintesi di ATP.

In questo processo, la perdita di elettroni dalla catena respiratoria induce la conversione dell'ossigeno $(0.4-4 \%)$ in radicali superossido. Di conseguenza, i mitocondri sono la fonte primaria di specie reattive dell'ossigeno (ROS).

Recenti scoperte sottolineano il coinvolgimento dei mitocondri in diversi disturbi renali e nella progressione del danno renale cronico (1). In particolare, il nostro gruppo di ricerca ha dimostrato una notevole disregolazione di un certo numero di elementi di OXPHOS, una down-regolazione dei componenti chiave del complesso mitocondriale IV (COXI e COXIV) e una significativa riduzione dell'attività del complesso $\mathrm{V}$ nei pazienti affetti da insufficienza renale cronica rispetto ai soggetti sani.

Una disfunzione dell'apparato mitocondriale è stata da noi segnalata anche nei pazienti uremici trattati con dialisi peritoneale (PD). 
Un gruppo di geni che determinano lo sviluppo mitocondriale (PGC-1 $\alpha$, NRF1 e TFAM) e che codificano per proteine mitocondriali costitutive (COX6C, COX7C, UQCRH e MCAD) è risultato down-regolato nei pazienti sottoposti a trattamento dialitico peritoneale rispetto ai controlli sani.

Inoltre, il fattore di trascrizione nucleare eritroide-2 (NRF2) e uno dei suoi geni bersaglio, la superossido dismutasi 2 (SOD2), sono risultati up-regolati nei pazienti in dialisi peritoneale, ponendo, quindi, l'ipotesi che esista un sistema antiossidante in grado di attivarsi per contrastare la produzione di ROS (2).

Anche a livello cardiaco, nel quadro della cardiomiopatia uremica, i mitocondri appaiono maggiormente sensibili allo stress ossidativo indotto da un danno ischemico e nel contempo la loro capacità di neutralizzare i ROS è notevolmente compromessa, come dimostrato nel modello murino di Taylor et al (3).

Inoltre in un altro modello murino di malattia mitocondriale (delezione di DNA mitocondriale) una sostanza di sintesi, l'acido mitochonico si è dimostrato efficace nel ridurre i livelli di ROS mitocondriali senza alterare l'attività dei complessi mitocondriali I-IV, sia a livello renale che cardiaco, dimostrando indirettamente come l'utilizzo di sostanze con target mitocondriale potrebbe favorire un rallentamento non solo della malattia renale cronica, ma anche della cardiomiopatia ad essa associata (4).

\section{Mitocondri: ruolo nella cronicizzazione del danno renale acuto}

Il rene è uno degli organi a maggior contenuto di mitocondri, avendo esso la necessità di molta energia pronta sotto forma di ATP, per il suo corretto funzionamento. In caso di danno renale acuto i mitocondri si rigonfiano, la loro funzionalità viene meno, contribuendo così alla cronicizzazione del danno. I meccanismi principalmente coinvolti in questo processo patogenetico sono la riduzione della produzione di ATP, il rilascio di proteine pro-apoptotiche nel citoplasma cellulare, l'incremento della produzione di ROS e l'incapacità mitocondriale di neutralizzarli.

Nell'ambito delle forme pre-renali di insufficienza renale acuta, quella secondaria a sepsi è caratterizzata da shock, infiammazione con attivazione citochinica e aggregazione piastrinica, più l'eventuale danno tossico indotto da antibiotici e/o mezzi di contrasto. La vacuolizzazione delle cellule tubulari renali descritta alla microscopia elettronica in questo contesto clinico è data proprio dalla presenza di mitocondri rigonfi e danneggiati, processo noto con il nome di fissione mitocondriale $(5,6)$.

La riparazione del danno renale acuto e la conseguente ripresa della funzione renale vanno di pari passo con il recupero della funzione mitocondriale ed in particolare con l'aumento dell'espressione di PGC1 $\alpha$, il fattore di trascrizione nucleare responsabile della rigenerazione mitocondriale, come dimostrato in un modello murino da Tran et al (7).
Stress ossidativo mitocondriale: un nuovo bersaglio terapeutico nella malattia renale cronica

I mitocondri potrebbero diventare, in futuro, un bersaglio farmacologico prezioso per i pazienti con insufficienza renale. Una varietà di agenti, in combinazione con le terapie convenzionali e uno stile di vita adeguato, avendo come target lo stress ossidativo mitocondrio-correlato, potrebbe prevenire e rallentare la progressione della malattia renale cronica e ridurre al minimo lo sviluppo di gravi complicanze sistemiche.

Tuttavia, anche se le proprietà antiossidanti della maggior parte di questi agenti sono ben note, il loro utilizzo in nefrologia clinica è stato solo parzialmente indagato.

Inoltre molti estratti naturali di piante sono di per sé nefrotossici e in tal senso studi più approfonditi di tossicità sistemica e renale, dose e non dose dipendente, saranno necessari e sempre più specifici grazie all'utilizzo delle scienze omiche, le quali potranno anche contribuire alla personalizzazione del trattamento (nutrigenomica e farmacogenomica) (8).

Al momento, gli agenti antiossidanti disponibili con un noto target mitocondriale sono: antiossidanti endogeni e derivati dagli alimenti, molecole con uno specifico target mitocondriale ed estratti naturali di piante (Fig. 1). Queste ultime sostanze sono state poco descritte in letteratura.

Pertanto, nella nostra revisione ci soffermeremo sulla descrizione delle potenzialità terapeutiche di questa importante categoria di prodotti naturali, che potrebbero rivestire, in futuro, un ruolo importante come antiossidanti nei pazienti con MRC.

\section{Estratti naturali di piante}

\section{Nigella sativa}

Nigella sativa (o cumino nero) è una pianta erbacea che cresce soprattutto nella zona mediterranea e in India, in gran parte utilizzata per fini culinari e medicinali (trattamento di disturbi polmonari, malattie cardiovascolari, febbre e influenza). Gli effetti biologici dei semi di Nigella sativa sembrano essere legati al suo olio, che contiene una quantità elevata di polifenoli e tocoferoli. Il timoquinone (TQ) e i suoi derivati (ditimoquinone, timoidroquinone e timolo) sono i più abbondanti.

La loro struttura chimica conferisce a queste molecole una significativa attività antiossidante come scavenger di ROS superossido, radicale idrossido e ossigeno singoletto (9). Inoltre, il TQ esercita proprietà anti-infiammatorie inibendo la produzione di ecosanoidi, trombossano B2 e leucotrieni B4 (10).

In medicina, il TQ e i suoi derivati sono stati testati come antiossidanti mitocondrio-mirati (11) e la Nigella sativa è stata impiegata come agente preventivo nei confronti della nefrotossicità indotta da numerosi agenti come, per esempio, la vancomicina (12). È stata utilizzata, inoltre, in corso di sindrome nefrosica per il controllo proteinuria e iperlipidemia (13).

Tuttavia, ulteriori studi sono necessari per valutarne gli effetti nella pratica clinica. 


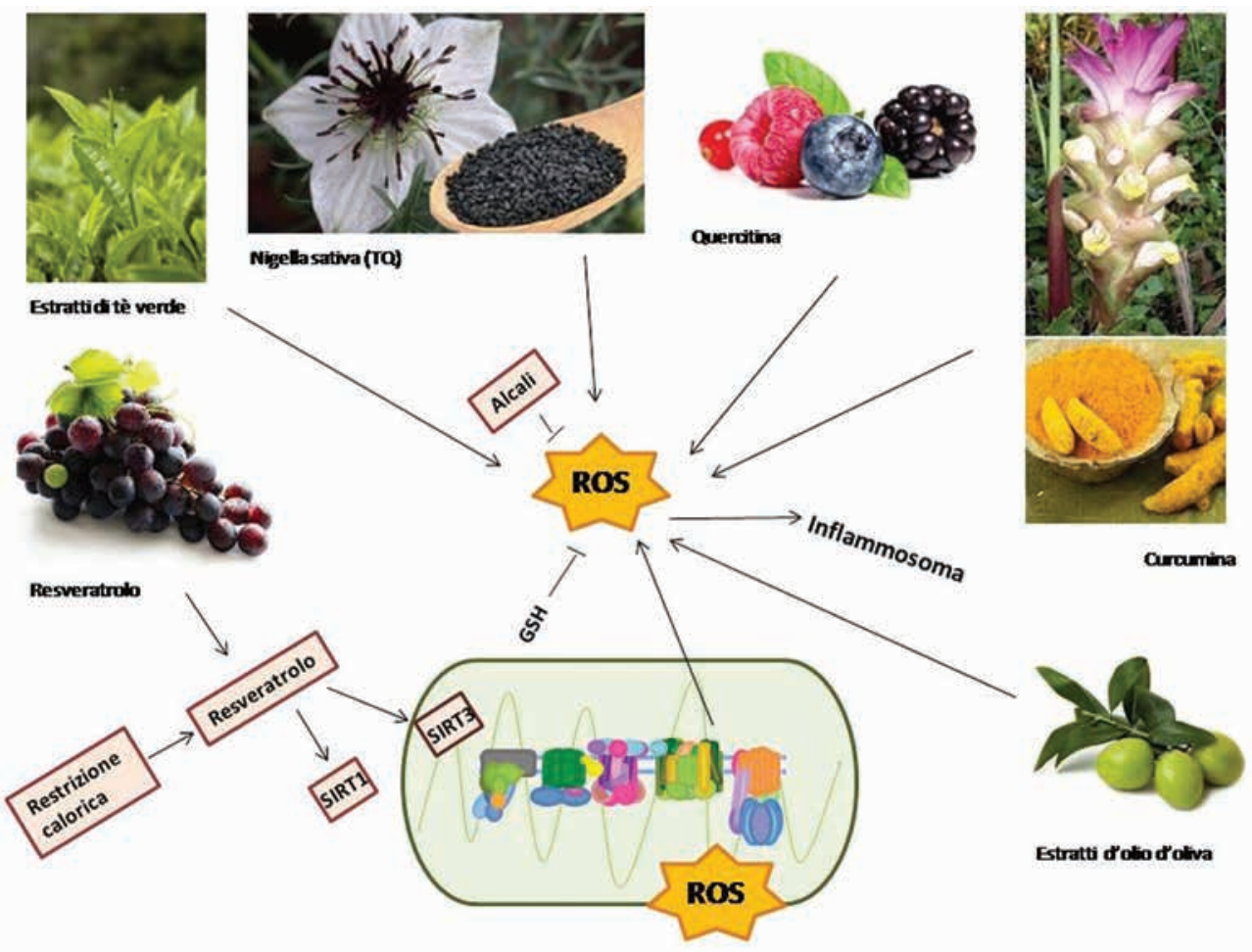

Fig. 1 - Azione dei principali fitoderivati con potenziale terapeutico nella MRC.

\section{Curcumina}

La curcuma è una spezia asiatica tradizionale derivata dall'omonimo rizoma della stessa famiglia dello zenzero (Zingiberacee).

È usata da secoli nella medicina tradizionale asiatica (ayurveda, medicina cinese, medicina araba). Recentemente, un gran numero di studi ha dimostrato che la curcumina, il principale curcuminoide contenuto nella Curcuma longa, esibisce proprietà altamente antiossidanti, anti-infiammatorie e antibatteriche.

Inoltre, come risultato in vari studi in vivo su modelli animali, la curcumina ha dimostrato potenziali effetti terapeutici renali. Attraverso le sue proprietà anti-infiammatorie, questo agente è stato in grado di ridurre la glomerulosclerosi e la fibrosi tubulo-interstiziale in un modello murino di CKD (ratti nefrectomizzati per 5/6) (14), di proteggere dalla progressione dell'insufficienza renale in modelli murini di nefropatia diabetica (15) e di ridurre al minimo la prevalenza di insufficienza renale in animali esposti a farmaci nefrotossici (per esempio, cisplatino) (16).

Gli effetti renoprotettivi riportati sono dovuti soprattutto ai seguenti fenomeni:

- $\quad$ inibizione di NF-kB (17);

- aumento della traslocazione nucleare di NRF2 con conseguente aumento della produzione di enzimi antiossidanti (come SOD, CAT, glutatione perossidasi) (18);

- $\quad$ riduzione dei livelli del recettore di tipo due per il TGFbeta, con conseguente riduzione della fosforilazione di SMAD2/3 (19);
- $\quad$ soppressione della sintesi di prostaglandine (PG) attraverso l'inibizione dell'espressione di mRNA della ciclossigenasi 2 e della lipossigenasi (20).

Inoltre, la curcumina è in grado di indurre una protezione cardiovascolare contro il rimodellamento cardiaco associato alla malattia renale cronica. Questo è dovuto in parte alla sua azione che preserva la funzione mitocondriale (21).

\section{Quercetina}

La quercetina (nomenclatura IUPAC: 3, 3', 4', 5.7-pentaidrossiflavanone) è un flavonolo presente in diversi alimenti (per esempio, cipolle, scalogno, mele, frutti di bosco, uva, capperi, brassicacee, tè e vino rosso) con forti proprietà antiossidanti, tra cui la capacità di catturare i radicali liberi, l'inibizione della xantina ossidasi e il decremento della perossidazione lipidica (22).

Esercita effetti adiuvanti nelle terapia del cancro, delle malattie cardiovascolari, dell'infiammazione sistemica e delle patologie gastrointestinali (23).

Ultimamente, è stato suggerito che la quercetina possa prevenire i danni ossidativi ai tessuti e attenuare il danno renale indotto dalla streptozotocina nei ratti diabetici (24).

Inoltre, Davis et al. hanno riportato che la supplementazione di quercetina per 7 giorni nei topi induce un aumento di mRNA di PGC-1 $\alpha$ e SIRT1, una maggiore resistenza del mtDNA e una maggiore concentrazione del citocromo c (25).

Esercita, inoltre, effetti anti-infiammatori inibendo NF$\mathrm{KB}$, modulando la sintesi di NOS e COX-2 e riducendo la pro- 
duzione di PCR (26).

La quercetina è stata somministrata in associazione con la curcumina in uno studio clinico volto a migliorare l'outcome dei riceventi un trapianto di rene da donatore cadavere. Questi bioflavonoidi hanno migliorato l'outcome, probabilmente attraverso l'induzione dell'eme ossigenasi-1 (27).

\section{Resveratrolo}

Il resveratrolo (3.5.4'-triidrossistilbene) è un fenolo naturale contenuto nel vino rosso e in alcuni alimenti, come I'uva, le arachidi e le bacche. Ha proprietà antiossidanti, antiinfiammatorie, anti-mutagene e antitumorali.

Questo fitocomposto esercita molte azioni differenti: ha un effetto antiossidante diretto, catturando e neutralizzando le ROS e inducendo l'espressione di diversi enzimi antiossidanti come SOD e CAT e attraverso l'induzione di NRF-2 (28); inibisce entrambe le attività della 5-lipossigenasi (LOX) e della ciclossigenasi (COX), con conseguente riduzione della produzione di mediatori infiammatori (29); inibisce la via del TGF- $\beta 1 /$ Smad3 e, quindi, riduce la fibrosi renale in modelli di ostruzione ureterale unilaterale $(30,31)$ e in ratti nefrectomizzati per 5/6 (32).

Infine, influenzando le attività delle MAPK, il resveratrolo impedisce la transizione epitelio-mesenchimale (EMT) indotta dal glucosio nelle cellule tubulari renali (33).

È stato dimostrato, in modelli animali, che questo fenolo può antagonizzare il danno renale acuto dovuto al cisplatino, il danno da ischemia-riperfusione e il danno indotto dalla sepsi (34-36).

Inoltre in modelli murini di glomerulonefrite membranosa, sindrome nefrosica, nefropatia diabetica e danno renale indotto da aldosterone, il resveratrolo ha dimostrato un efficace effetto anti-proteinurico. In questo contesto, oltre ai meccanismi d'azione già riportati, il resveratrolo è in grado di attivare una sirtuina, SIRT1 che porta all'aumento dell'espressione di PGC- $1 \alpha$, gene che controlla la biogenesi e la funzione mitocondriale (37-40).

Purtroppo, il resveratrolo presenta una scarsa biodisponibilità, rendendo difficile tradurre i suddetti risultati in vitro in studi clinici (41). Al momento, ne sono in corso alcuni su diverse malattie sistemiche metaboliche e infiammatorie.

\section{Polifenoli del tè verde}

I principali polifenoli presenti nel tè verde sono epigallocatechina 3-O-gallato, epicatechina 3-0-gallato, epigallocatechina ed epicatechina. Le azioni benefiche delle catechine sono dovute principalmente alle proprietà antiossidanti (capacità di scavenging delle ROS) e alla capacità di chelare gli ioni metallici quali il rame (II) e il ferro (III) e di formare il radicale libero stabile semichinone (42). L'epigallocatechina-3-gallato (EGCG) è il più abbondante e più attivo polifenolo presente nel tè verde.
Diversi meccanismi sono stati associati alle proprietà antiinfiammatorie dell'EGCG, come: a) l'inibizione di NFkB (43) e b) l'attivazione di AMPK che inibiscono la produzione di numerosi mediatori proinfiammatori inclusi TNF- $\alpha$, IL-1 $\beta$, IL-6, MCP1 (monocyte chemoattractant protein-1), ossido nitrico sintasi inducibile (iNOS) e ciclossigenasi-2, in seguito alla stimolazione con LPS (44-46). Più recentemente, Qin et al. hanno anche riportato un'interazione diretta tra EGCG e chemochine, con una conseguente limitazione dei loro effetti biologici (47).

Nei ratti sottoposti a ostruzione ureterale unilaterale, la somministrazione di EGCG ha causato un'up-regolazione e traslocazione nucleare di NRF2 con conseguente aumento dell'espressione degli enzimi antiossidanti, come glutatione perossidasi, glutatione S-transferasi, $\mathrm{\gamma}$-GCS ed eme ossigenasi-1 (48).

Allo stesso tempo, l'EGCG allevia il danno glomerulare e tubulare e attenua la fibrosi interstiziale renale inibendo lapathway di TGF- $\beta /$ Smad e l'up-regolazione di NFkB (49).

Nei ratti Wistar sottoposti a danno renale da ischemia-riperfusione con iniezione di LPS, la somministrazione di EGCG riduce l'attività della mieloperossidasi e protegge il rene dal danno indotto dal radicale perossinitrito (50).

Polifenoli del tè verde $(40 \mathrm{mg} / \mathrm{die})$ somministrati per 6 mesi a 50 pazienti in emodialisi hanno diminuito i livelli di metilguanidina (MG), una tossina uremica prodotta dallo stress ossidativo, grazie alla loro capacità di scavenging delle ROS (51).

Inoltre, queste sostanze possono proteggere i reni dal danno indotto da vari farmaci nefrotossici come, per esempio, la ciclosporina (52).

\section{Estratti di olio d'oliva}

I polifenoli contenuti nell'olio di oliva sono noti per le loro attività antiossidanti e protettive. In particolare, il fenolo idrossitirosolo esercita un'attività regolatoria della funzione mitocondriale. In uno studio di Cao et al., topi C57BL/6J sono stati nutriti con una dieta ad alto contenuto di grassi, supplementata con idrossitirosolo (HT) per 17 settimane. Dopo 17 settimane, la supplementazione con HT si è dimostrata in grado di far regredire l'accumulo adiposo nel tessuto muscolare scheletrico e a livello epatico. I livelli di attività mitocondriale sono risultati aumentati sia direttamente che indirettamente, mediante il riscontro di ridotti livelli di proteine e di lipidi ossidati. Infine, HT ha portato a un'importante riduzione della glicemia a digiuno e del profilo lipidico. L'azione mitocondriale dell'HT potrebbe rivelarsi altrettanto importante nella malattia renale cronica, spesso associata a sindrome metabolica e gravata da un'importante disfunzione mitocondriale (53).

\section{Dieta mediterranea, microbiota e MRC}

Numerose sostanze riportate in questo articolo sono tipicamente presenti nelle dieta tradizionale dei paesi mediterra- 
nei, con qualche peculiarità locale.

La dieta mediterranea è caratterizzata da un'elevata assunzione di frutta e verdura, legumi, cereali e olio d'oliva e da uno scarso consumo di proteine animali, le quali sono prevalentemente di origine ittica, associati a un moderato consumo di vino.

In molti studi di popolazione, sia europei che statunitensi (studio ATTICA, studio The Northern Manhattan Study NO$M A S)$, un regime dietetico di tipo mediterraneo si è dimostrato associato alla presenza di una migliore funzione renale, nonché efficace nel prevenire l'insufficienza renale stessa, il rischio cardiovascolare ad essa associato, l'insorgenza di numerose neoplasie e la mortalità globale $(54,55)$. È interessante notare che una dieta di tipo mediterraneo si è dimostrata efficace nelle prevenzione della malattia renale cronica e nel ridurre la mortalità correlata, anche in popolazioni lontane dal bacino mediterraneo, come nel caso della popolazione svedese e in popolazioni multietniche $(55,56)$, eliminando, così, possibili bias ambientali.

La dieta mediterranea, grazie al basso contenuto di grassi saturi e di sodio e all'elevato contenuto di antiossidanti, protegge, quindi, l'endotelio ed esercita un'azione anti-infiammatoria; migliora, inoltre, l'emodinamica renale mimando l'azione di un vero e proprio ACE-inibitore o di un antagonista del recettore dell'angiotensina II (sartano) (55).

Inoltre, il moderato consumo di vino caratteristico della dieta mediterranea spiega in parte il famoso "paradosso francese" secondo il quale il vino rosso, ricco di resveratrolo, riduce le malattie cardiovascolari anche in presenza di una dieta ad alto contenuto di grassi (57).

Più recentemente, dei gruppi italiani hanno dimostrato la presenza di antiossidanti efficaci anche nel vino bianco; esso, infatti, pur non contenendo dei polifenoli, contiene, invece, dei fenoli semplici, come il tirosolo e l'idrossitirosolo (caratteristicamente presenti nell'olio d'oliva) e l'acido caffeico, che, anche a bassi dosaggi, modulando il rilascio di ossido nitrico, esercita un'azione protettiva sull'endotelio, rallentando, così, la progressione del danno cardiaco e renale $(58,59)$.

Infine, negli ultimi anni, è apparso evidente che l'alto contenuto di fibre presenti in frutta, verdura e cereali integrali promuove la crescita di una flora batterica saccarolitica (Bifidobatteri, Lattobacilli), piuttosto che proteolitica. La conversione da parte di questi ceppi dei carboidrati complessi in acidi grassi a catena corta (SCA) esercita un'azione protettiva sugli enterociti e un'azione immunomodulante e anti-infiammatoria sistemica.

Nel paziente con insufficienza renale cronica, il microambiente intestinale è, di per sé, alterato per l'aumentata escrezione intestinale di acido urico, urea e ossalati, che promuovono la crescita di una flora batterica proteolitica con produzione di indoli e fenoli, i quali sono tossine uremiche che peggiorano la progressione del danno renale. L'alterata permeabilità intestinale favorisce, infine, la traslocazione bat- terica, l'endotossinemia e l'infiammazione cronica. Appare, dunque, evidente come la dieta mediterranea promuova anche una minor sintesi di tossine uremiche, rallentando, così, la progressione del danno renale (60).

Nel suo complesso per l'azione antiossidante, anti-infiammatoria e modulante la flora batterica intestinale, la dieta mediterranea appare la dieta migliore per il paziente affetto da MRC.

\section{Conclusioni}

Una varietà di estratti naturali derivati da piante potrebbe prevenire e adiuvare il trattamento della malattia renale cronica, rallentandone la progressione.

Tuttavia, il loro impiego nella pratica clinica è stato solo parzialmente valutato e il corretto utilizzo degli stessi deve essere ancora ben definito in nefrologia. Infatti, anche se gli effetti positivi di questi composti sono ben noti, sono ancora necessari ampi studi in vivo, su modelli animali, e successivi studi clinici per fornire informazioni maggiormente attendibili sulla loro efficacia nella malattia renale cronica.

Una serie di strategie future, compreso lo studio della farmacogenomica e della nutrigenomica, dovrebbe essere intrapresa per identificare i pazienti potenzialmente sensibili ai trattamenti antiossidanti mitocondrio-mirati, personalizzandoli.

In attesa che queste evidenze acquisiscano solidità appare ragionevole incoraggiare uno stile di vita e uno stile alimentare adeguati che migliorino l'efficienza mitocondriale nei pazienti con malattia renale cronica. Per raggiungere questo obiettivo, la dieta mediterranea, ricca di numerosi antiossidanti, alcuni dei quali menzionati in questa review, potrebbe rappresentare un valido strumento.

\section{Glossario}

- $\quad$ ATP: adenosina trifosfato, molecola trasportatrice di energia della cellula, formata da una base azotata, l'adenina, da uno zucchero, il ribosio, e da tre gruppi fosfato.

- $\quad$ CAT: catalasi, enzima appartenente alla classe delle ossido-reduttasi, coinvolto nella detossificazione della cellula da specie reattive dell'ossigeno.

- $\quad$ CKD (chronic kidney disease): insufficienza renale cronica.

- COX-2: ciclossigenasi 2, una delle forme isoenzimatiche della prostaglandina-endoperossido sintasi; al contrario della COX-1, che è un enzima costitutivo, la COX-2 è un enzima inducibile ed è presente solo durante i processi infiammatori nei tessuti colpiti da infiammazione.

- COXI, COX IV: complessi mitocondriali I e IV.

- COX6C: sub unità 6C della citocromo c ossidasi, enzima terminale della catena respiratoria; catalizza il trasferimento di elettroni dal citocromo c all'ossigeno molecolare, che viene ridotto a due molecole d'acqua.

- $\quad$ COX7C: sub unità 7C della citocromo $\mathrm{c}$ ossidasi, enzima terminale della catena respiratoria; catalizza il trasferimento di elet- 
troni dal citocromo $\mathrm{c}$ all'ossigeno molecolare, che viene ridotto a due molecole d'acqua.

- EGCG: epigallocatechina gallato, la catechina più abbondante nel tè e, in particolare, nel tè verde.

- EMT: transizione epiteliale-mesenchimale, processo in cui, in seguito a uno stimolo cronico, le cellule epiteliali con polarità basale-apicale perdono il loro fenotipo e acquisiscono le caratteristiche delle cellule mesenchimali non polarizzate.

- FADH2: flavin adenine dinucleotide 2, molecola coinvolta nel trasferimento degli elettroni, quindi nelle reazioni di ossidoriduzione.

- HT: idrossitirosolo, composto fitochimico antiossidante, derivato dall'ulivo.

- IL-1 $\beta$ : interleuchina $1 \beta$, citochina secreta da vari tipi di cellule del sistema immunitario tra cui macrofagi, monociti e cellule dendritiche, ma anche da fibroblasti e cellule endoteliali.

- $\quad$ iNOS: sintetasi inducibile dell'ossido nitrico, enzima per la produzione di radicali liberi nei macrofagi attivati, assente nel citosol dei macrofagi quiescenti, ma la cui sintesi può essere rapidamente indotta per l'azione combinata di LPS e INF-gamma.

- LOX: lipossigenasi, un enzima appartenente alla classe delle ossidoreduttasi che produce idroperossidi coniugati attraverso l'ossidazione di acidi grassi polinsaturi. Esso catalizza la prima reazione della cosiddetta via della lipossigenasi, coinvolta nella risposta dell'organismo ai traumi e agli stress esterni.

- $\quad$ LPS: lipopolisaccaride, uno dei componenti della parete cellulare dei batteri Gram-negativi, endotossina che provoca un'intensa risposta da parte dell'organismo, con febbre, vasodilatazione e shock.

- MAPK: MAP-chinasi (proteina attivata da mitogeni), ultima chinasi attivata nella via Ras/Raf, che fosforila uno o più fattori di trascrizione genica, portando a varie risposte cellulari, tra cui la divisione cellulare.

- MCAD (medium-chain acyl-CoA dehydrogenase): isoforma dell'acil-CoA deidrogenasi, coinvolta nell'ossidazione degli acidi grassi a catena media.

- MCP-1 (Monocyte Chemoattractant Protein-1): nota anche con I'acronimo CCL2, citochina coinvolta nel reclutamento di diversi tipi di leucociti.

- MRC: malattia renale cronica.

- NADH: nicotinammide adenina dinucleotide, molecola coinvolta nel trasferimento degli elettroni, quindi nelle reazioni di ossido-riduzione.

- NRF1: fattore respiratorio nucleare-1, fattore di trascrizione di geni coinvolti nel metabolismo cellulare e nella trascrizione e nella replicazione del DNA mitocondriale.

- NRF2: fattore di trascrizione nucleare eritroide-2, fattore che regola l'espressione genica di una grande varietà di enzimi citoprotettivi antiossidanti.

- NF-kB (nuclear factor kappa-light-chain-enhancer of activated $B$ cells): fattore di trascrizione coinvolto in tutti i tipi di cellule in risposta a stress, citochine, radicali liberi e antigeni batterici o virali.
- OXPHOS: fosforilazione ossidativa, ovvero il processo cellulare che avviene nei mitocondri per la produzione di ATP (adenosina trifosfato).

- PD: dialisi peritoneale.

- $\quad$ PGC-1 $\alpha$ : gene che codifica per il coattivatore-1 $\alpha$ dei recettori gamma attivati dai proliferatori dei perossisomi, un recettore coinvolto nel metabolismo lipidico e dei carboidrati e nella sensibilità all'insulina.

- $\quad$ PG: prostaglandine, acidi ciclopentanoici derivati dall'acido arachidonico, che rivestono un ruolo biologico importante come mediatori flogistici.

- $\quad$ ROS: specie reattive dell'ossigeno.

- $\quad \mathrm{SMAD2/3}$ (small mother against decapentaplegic): classe di proteine che modulano l'attività dei ligandi del fattore di crescita trasformante beta, penetrano nel nucleo e fungono da fattori di trascrizione.

- $\quad$ SIRT1: sirtuina 1, enzima che deacetila le proteine che contribuiscono alla regolazione cellulare in risposta a fattori di stress.

- SOD2: superossido dismutasi 2, isoforma mitocondriale. Proteina antiossidante cellulare, che neutralizza l'anione superossido e lo converte in idrogeno perossido e ossigeno bivalente.

- TFAM: gene che codifica per il fattore di trascrizione mitocondriale $A$, fattore chiave di attivazione della trascrizione e replicazione del DNA mitocondriale.

- TQ: timochinone, molecola antiossidante ricavata da Nigella sativa.

- $\quad$ TGF- $\beta$ (transforming growth factor beta): fattore di crescita trasformante, citochina coinvolta nel controllo della proliferazione e della differenziazione cellulare.

- TNF- $\alpha$ : fattore di necrosi tumorale $\alpha$, citochina coinvolta nell'infiammazione sistemica.

- UQCRH: componente del complesso dell'ubichinolo-citocromo $C$ reduttasi, parte della catena respiratoria mitocondriale.

\section{Disclosures}

Financial support: No financial support was received for this submission.

Conflict of interest: The authors have no conflict of interest.

\section{Bibliografia}

1. Granata S, Zaza G, Simone S, et al. Mitochondrial dysregulation and oxidative stress in patients with chronic kidney disease. BMC Genomics 2009;10:388.

2. Zaza G, Granata S, Masola V, et al. Downregulation of nuclearencoded genes of oxidative metabolism in dialyzed chronic kidney disease patients. PLoS One 2013;8(10):e77847.

3. Taylor D, Bhandari S, Seymour AM. Mitochondrial dysfunction in uremic cardiomyopathy. Am J Physiol Renal Physiol 2015;308(6):F579-87.

4. 4. Suzuki T, Yamaguchi H, Kikusato M, et al. Mitochonic acid 5 binds mitochondria and ameliorates renal tubular and cardiac myocyte damage. J Am Soc Nephrol. 2015. [Epub ahead of print]

5. Yu Ishimoto and Reiko Inagi. Mitochondria: a therapeutic target in acute kidney injury. Nephrol Dial Transplant.2015. [Epub ahead of print] 
6. Parikh SM. Therapeutic targeting of the mitochondrial dysfunction in septic acute kidney injury. Curr Opin Crit Care 2013;19(6):554-9.

7. Tran M, Tam D, Bardia A, et al. PGC-1 $\alpha$ promotes recovery after acute kidney injury during systemic inflammation in mice. J Clin Invest 2011;121(10):4003-14.

8. Ouedraogo M, Baudoux T, Stévigny $C$, et al. Review of current and "omics" methods for assessing the toxicity (genotoxicity, teratogenicity and nephrotoxicity) of herbal medicines and mushrooms. J Ethnopharmacol 2012;140(3):492-512.

9. Badary OA, Taha RA, Gamal el-Din AM, Abdel-Wahab MH. Thymoquinone is a potent superoxide anion scavenger. Drug Chem Toxicol 2003;26:87-98.

10. Houghton PJ, Zarka R, de las Heras B, Hoult JR. Fixed oil of Nigella sativa and derived thymoquinone inhibit eicosanoid generation in leukocytes and membrane lipid peroxidation. Planta Med 1995;61:33-6.

11. Severina II, Severin FF, Korshunova GA, et al. In search of novel highly active mitochondria-targeted antioxidants: thymoquinone and its cationic derivatives. FEBS Lett 2013;587:2018-24.

12. Basarslan F, Yilmaz N, Ates S, et al. Protective effects of thymoquinone on vancomycin-induced nephrotoxicity in rats. Hum Exp Toxicol 2012;31:726-33.

13. Badary $\mathrm{OA}$, Abdel-Naim $\mathrm{AB}$, Abdel-Wahab $\mathrm{MH}$, Hamada FM. The influence of thymoquinone on doxorubicininduced hyperlipidemic nephropathy in rats. Toxicology 2000;143(3):219-26.

14. Tapia E, Soto V, Ortiz-Vega KM, et al. Curcumin induces Nrf2 nuclear translocation and prevents glomerular hypertension, hyperfiltration, oxidant stress, and the decrease in antioxidant enzymes in 5/6 nephrectomized rats. Oxid Med Cell Longev 2012;2012:269039.

15. Sharma S, Kulkarni SK, Chopra K. Curcumin, the active principle of turmeric (Curcuma longa), ameliorates diabetic nephropathy in rats. Clin Exp Pharmacol Physiol 2006;33:940-5.

16. Antunes LM, Darin JD, Bianchi Nde L. Effects of the antioxidants curcumin or selenium on cisplatin-induced nephrotoxicity and lipid peroxidation in rats. Pharmacol Res 2001;43:145-50.

17. Singh SS, Aggarwal BB. Activation of transcription factor NF-kB is suppressed by curcumin (diferuloylmethane). J Biol Chem 1995;270:24995-5000. J Biol Chem.1995;270(42):24995-25000.

18. Soetikno V, Sari FR, Lakshmanan AP, et al. Curcumin alleviates oxidative stress, inflammation, and renal fibrosis in remnant kidney through the Nrf2-keap1 pathway. Mol Nutr Food Res 2013;57:1649-59.

19. Gaedeke J, Noble NA, Border WA. Curcumin blocks multiple sites of the TGF-beta signaling cascade in renal cells. Kidney Int 2004;66(1):112-20.

20. Rao CV. Regulation of COX and LOX by curcumin. Adv Exp Med Biol 2007;595:213-26.

21. Correa F, Buelna-Chontal $M$, Hernández-Reséndiz $S$, et al. Curcumin maintains cardiac and mitochondrial function in chronic kidney disease. Free Radic Biol Med 2013;61:119-29.

22. Morand C, Crespy V, Manach C, Besson C, Demigne C, Remesy C. Plasma metabolites of quercetin and their antioxidant properties. Am J Physiol 1998;75:212-9.

23. Middleton E Jr. Effect of plant flavonoids on immune and inflammatory cell function. Adv Exp Med Biol 1998;439:175-82.

24. Sanders RA, Rauscher FM, Watkins III JB. Effects of quercetin on antioxidant defence in streptozotocin-induced diabetic rats. J Biochem Mol Toxicol 2001;15:143-9.

25. Davis JM, Murphy EA, Carmichael MD, Davis B. Quercetin increases brain and muscle mitochondrial biogenesis and exercise tolerance. Am J Physiol Regul Integr Comp Physiol 2009;296:1071-7.
26. Min YD, Choi $\mathrm{CH}$, Bark $\mathrm{H}$, et al. Quercetin inhibits expression of inflammatory cytokines through attenuation of NF-KB and p38 MAPK in HMC-1 human mast cell line. Inflamm Res 2007; 56:210-5.

27. Shoskes D, Lapierre C, Cruz-Correa M, et al. Beneficial effects of the bioflavonoids curcumin and quercetin on early function in cadaveric renal transplantation: a randomized placebo controlled trial. Transplantation 2005;80:1556-9.

28. Mokni M, Elkahoui S, Limam F, Amri M, Aouani E. Effect of resveratrol on antioxidant enzyme activities in the brain of healthy rat. Neurochem Res 2007;32:981-7.

29. Kimura Y, Okuda, H, Arichi S. Effects of stilbenes on arachidonate metabolism in leukocytes. Biochim Biophys Acta 1985;834: 275-8.

30. Li J, Qu X, Ricardo SD, Bertram JF, Nikolic-Paterson DJ. Resveratrol inhibits renal fibrosis in the obstructed kidney: potential role in deacetylation of Smad3. Am J Pathol 2010;177: 1065-71.

31. Liang J, Tian S, Han J, Xiong P. Resveratrol as a therapeutic agent for renal fibrosis induced by unilateral ureteral obstruction. Ren Fail 2014;36:285-91.

32. Huang $X Z$, Wen $D$, Zhang $M$, et al. Sirt1 activation ameliorates renal fibrosis by inhibiting the TGF- $\beta / S$ mad3 pathway. J Cell Biochem 2014;115:996-1005.

33. He T, Guan X, Wang S, et al. Resveratrol prevents high glucoseinduced epithelial-mesenchymal transition in renal tubular epithelial cells by inhibiting NADPH oxidase/ROS/ERK pathway. Mol Cell Endocrinol 2015;402:13-20.

34. Do Amaral CL, Francescato HD, Coimbra TM, et al. Resveratrol attenuates cisplatin-induced nephrotoxicity in rats. Arch Toxicol 2008;82:363-70.

35. Bertelli AA, Migliori $M$, Panichi $V$, et al. Resveratrol, a component of wine and grapes, in the prevention of kidney disease. Ann NY Acad Sci 2002;957:230-8.

36. Holthoff $\mathrm{JH}$, Wang Z, Seely KA, et al. Resveratrol improves renal microcirculation, protects the tubular epithelium, and prolongs survival in a mouse model of sepsis-induced acute kidney injury. Kidney Int 2012;81:370-8.

37. Wu CC, Huang YS, Chen JS, et al. Resveratrol ameliorates renal damage, increases expression of heme oxygenase- 1 , and has anti-complement, anti-oxidative, and anti-apoptotic effects in a murine model of membranous nephropathy. PLoS One 2015;10(5):e0125726.

38. Nihei T, Miura Y, Yagasaki K. Inhibitory effect of resveratrol on proteinuria, hypoalbuminemia and hyperlipidemia in nephritic rats. Life Sci 2001;68(25):2845-52.

39. Yuan $Y$, Huang $S$, Wang $W$, et al. Activation of peroxisome proliferator-activated receptor- $\psi$ coactivator $1 \alpha$ ameliorates mitochondrial dysfunction and protects podocytes from aldosterone-induced injury. Kidney Int 2012;82(7):771-89.

40. Sharma S, Anjaneyulu M, Kulkarni SK, Chopra K. Resveratrol, a polyphenolic phytoalexin, attenuates diabetic nephropathy in rats. Pharmacology 2006;76(2):69-75.

41. Smoliga JM, Blanchard O. Enhancing the delivery of resveratrol in humans: if low bioavailability is the problem, what is the solution? Molecules 2014;19:17154-72.

42. Higdon JV, Frei B. Tea catechins and polyphenols: health effects, metabolism, and antioxidant functions. Crit Rev Food Sci Nutr 2003;43:89-143.

43. Sarkar FH, Li Y, Wang Z, Kong D. Cellular signaling perturbation by natural products. Cell Signal 2009;21:1541-7.

44. Ahn HY, Xu Y, Davidge ST. Epigallocatechin-3-O-gallate inhibits TNF alpha-induced monocyte chemotactic protein-1 production from vascular endothelial cells. Life Sci 2008;82:964-8.

45. Ahmed S, Marotte H, Kwan K, et al. Epigallocatechin-3-gallate 
inhibits IL-6 synthesis and suppresses transsignaling by enhancing soluble gp130 production. Proc Natl Acad Sci USA 2008;105:14692-7.

46. Tedeschi E, Menegazzi M, Yao $Y$, Suzuki H, Förstermann $U$, Kleinert $\mathrm{H}$. Green tea inhibits human inducible nitric-oxide synthase expression by down-regulating signal transducer and activator of transcription-1 $\alpha$ activation. Mol Pharmacol 2004;65:111-20.

47. Qin S, Alcorn JF, Craigo JK, et al. Epigallocatechin-3-gallate reduces airway inflammation in mice through binding to proinflammatory chemokines and inhibiting inflammatory cell recruitment. J Immunol 2011;186(6):3693-700.

48. Zhou P, Yu JF, Zhao CG, Sui FX, Teng X, Wu YB. Therapeutic potential of EGCG on acute renal damage in a rat model of obstructive nephropathy. Mol Med Rep 2013;7:1096-102.

49. Wang Y, Wang B, Du F, et al. Epigallocatechin-3-Gallate Attenuates Unilateral Ureteral Obstruction-Induced Renal Interstitial Fibrosis in Mice. J Histochem Cytochem 2015;63(4):270-9.

50. Yokozawa T, Rhyu DY, Cho EJ. (-)-Epicatechin 3-O-gallate ameliorates the damages related to peroxynitrite production by mechanisms distinct from those of other free radical inhibitors. J Pharm Pharmacol 2004;56(2):231-9.

51. Yokozawa $\mathrm{T}$, Dong $\mathrm{E}$, Oura $\mathrm{H}$. Proof that green tea tannin suppresses the increase in the blood methylguanidine level associated with renal failure. Exp Toxicol Pathol 1997;49:117-22.

52. Rehman H, Krishnasamy Y, Haque K, et al. Green tea polyphenols stimulate mitochondrial biogenesis and improve renal function after chronic cyclosporin a treatment in rats. PLoS One 2013;8 (6):e65029.
53. Cao K, Xu J, Zou X, et al. Hydroxytyrosol prevents dietinduced metabolic syndrome and attenuates mitochondrial abnormalities in obese mice. Free Radic Biol Med 2014;67: 396-407.

54. Chrysohoou C, Panagiotakos DB, Pitsavos C, et al. Adherence to the Mediterranean diet is associated with renal function among healthy adults: the ATTICA study. J Ren Nutr 2010; 20(3):176-84.

55. Khatri $M$, Moon $\mathrm{YP}$, Scarmeas $\mathrm{N}$, et al. The association between a Mediterranean-style diet and kidney function in the Northern Manhattan Study cohort. Clin J Am Soc Nephrol 2014;9(11):1868-75.

56. Huang $X$, Jiménez-Moleón JJ, Lindholm B, et al. Mediterranean diet, kidney function, and mortality in men with CKD. Clin J Am Soc Nephrol 2013;8(9):1548-55.

57. Catalgol B, Batirel S, Taga Y, Ozer NK. Resveratrol: French paradox revisited. Front Pharmacol 2012;3:141.

58. Migliori M, Panichi V, de la Torre $\mathrm{R}$, et al. Anti-inflammatory effect of white wine in CKD patients and healthy volunteers. Blood Purif 2015;39(1-3):218-23.

59. Migliori M, Cantaluppi V, Mannari C, et al. Caffeic acid, a phenol found in white wine, modulates endothelial nitric oxide production and protects from oxidative stress-associated endothelial cell injury. PLoS One 2015;10(4):e0117530.

60. Montemurno E, Cosola C, Dalfino G, et al. What would you like to eat, Mr CKD Microbiota? A Mediterranean Diet, please! Kidney Blood Press Res 2014;39(2-3):114-23. 